\title{
Comparing Effectiveness of Soft, Medium and Hard Bristle Tooth Brushes on Oral Hygiene
}

\author{
Wafaa Abdelraouf Khalil \\ Assistant Professor of Human Physiology, Basic Sciences Department, Faculty of Dentistry, Khartoum University
}

\begin{abstract}
Background: With the increasing rate of oral diseases there is a progressive need for effective measures for prevention and provision of better oral health. Tooth brushes as a commercial and effective tools are available with different bristle textu res, sizes, and forms. This study aims to compare the effect of soft, medium and hard bristle tooth brushes on oral hygiene, using oral hygiene indices. Methods: Included in the study 59 regular brush male users recruited on voluntary basis, with a mean age of 39.03 \pm 10.34 . A face to face questionnaire was used to assess type of texture of their tooth brushes coupled with gingival examination. Excluded from the study those with chronic illnesses, on oral mouth wash or receiving antibiotics for the last one month. Results: Significant differences were detected in the oral hygiene measure Gingival index $(G I)$ when related to the three textures of the tooth paste brush bristles with a $p$-value $=0.001$ between soft and medium and 0.023 between soft and hard bristles. High gingival index was shown with the soft texture, and a significant difference in the Plaque index (PI) between soft and medium texture (p-value $=0.001)$. Conclusion: Best gingival index reported on using medium texture, while the worst index with the soft texture regardless of type of tooth brush used. Further research is, however, recommended.
\end{abstract}

Keywords: Oral hygiene indices, Gingival Index, Plaque Index, Toothbrush, Soft, Medium \& Hard Bristle Textures

\section{Introduction}

Dental caries and periodontal diseases exert tremendous social, economic, and financial burden on a global scale. Despite a plethora of preventive measures available, dental plaque, the precursor of dental caries and periodontal disease, remains an enigma ${ }^{(1)}$. Dental plaque is a sticky film of bacteria that can irritate the gums, harden into tartar, resulting in gingivitis, a swollen bleeding gum tissue. Dental plaque if effectively carried away, can be the most effective method of maintaining good oral hygiene, reducing tooth decay, and promoting better gingival health ${ }^{(1)}$. Of the various methods employed to maintain oral hygiene, tooth brushing is the most common one ${ }^{(3)}$, as considered an effective way of removing plaque, preventing gingivitis and also managing dental caries (4). However, the value of uncontrolled tooth brushing in prevention of dental caries has always been questioned ${ }^{(5)}$. Manual dexterity and motivation of an individual are of paramount importance in maintaining oral hygiene, and besides proper brushing technique, effective plaque removal depends also on the type of toothbrush ${ }^{(6)}$. Toothbrush design and materials have come a long way. Many factors are interplaying for maintaining a healthy oral hygiene. Loe et al. showed the essential role of dental plaque to the etiology of gingivitis, and that removal of plaque can reverse this process ${ }^{(7)}$. Design of the toothbrush, the skill of the individual using the brush, tooth brushing frequency, duration of use are pivotal factors in controlling plaque accumulation hence preventing gingivitis and/or periodontitis and decays effectively ${ }^{(8)}$. The last two factors represent individual tooth brushing behavior and are affected by learning experience, motivation and manual capacity and can of course be improved with good co-operation established between dentists and patients ${ }^{(9)}$. However, the first factor represent technology improvement and is affected by the physical and mechanical properties of the toothbrush bristles, shape, size and morphometry of the tooth brush heads and handles ${ }^{(10)}$. Furthermore, it was wellknown that most people use a simple horizontal tooth brushing action and brush their teeth for the duration markedly shorter than optimal time ${ }^{(11)}$. Manufacturers of toothbrushes aim for innovations in the brush head design that will help to compensate for non-ideal tooth brushing technique and time ${ }^{(12)}$. The more basic designs include tooth brushes with standard (straight) bristles and more advanced models with angled (CrissCross ${ }^{\circledR}$ ) bristles specially aiming at helping to remove plaque from teeth and along the gum line ${ }^{(13,14)}$. The advanced toothbrushes has the potential to remove greater amounts of plaque, especially from the gum lines and approximal surfaces than conventional tooth brushes incorporating straight bristles ${ }^{(15,16)}$. On the other hand, there has been conflicting results whether which design is more capable of effective plaque control ${ }^{(8-14)}$. First toothbrushes were developed solely to effectively remove plaque and they had hard and then medium bristle softness. Recently, soft bristles were used in straight and criss-cross brushes as hard and medium bristle stiffness could have the potential for causing soft tissue damage. Harder tooth brushes reduce plaque more efficiently but are more stressful to teeth and gum, while using a medium to soft tooth brush with a longer cleaning time was rated to be the best compromise between cleaning result and gum and tooth health ${ }^{(15)}$. Most dentists recommend using a soft toothbrush since hard bristled toothbrushes can damage tooth enamel and irritate the gums ${ }^{(16)}$. At a time when toothbrushes are over-the-counter products, no evidence based instruction for their use or renewal, neither of the benefits of one type bristle texture over the others, is available. Published scientific data comparing the effect of soft, medium and hard bristle tooth brushes on oral hygiene are so scarce. There is need for more research in this issue. In this parallel-group cross-sectional comparative study we are therefore aiming to bridge the gap by comparing the efficacy of plaque control and potential effects on gingival damage of the different toothbrush bristle textures. 


\section{International Journal of Science and Research (IJSR) \\ ISSN (Online): 2319-7064 \\ Index Copernicus Value (2015): 78.96 | Impact Factor (2015): 6.391}

\section{Subjects \& Methods}

Regular tooth brush users were selected through a voluntary non-probability sampling technique, interviewed using a structured questionnaire to assess their demographic profiles and oral hygiene habits, then subjected to oral clinical assessment. Regular brush users were defined as individuals who reported using tooth brushes of any type at least one time daily for the last year. Other inclusion criteria included good general health, absence of chronic illnesses such as diabetes and hypertension, no past or present history of smoking or smokeless tobacco (Tomback - oral snuffing/sniffing/dipping) use. Exclusion criteria included those who were in regular mouth wash use or antibiotic intake, caffeine containing drinks for more than once/day, within the last month before clinical assessment. Study population was comprised of employees and students from the Faculty of Medicine, Khartoum University, Sudan. Through a parallel-group cross-sectional comparative design, out of an initial 59 study participants interviewed and preliminary assessed clinically, 57 completed the research, who were then divided into three groups: those using hard brittle texture toothbrushes $(n=14)$, soft brittle texture toothbrushes ${ }^{(17)}$. (19), and medium brittle texture $(\mathrm{N}=24)$. Recruitment of study groups continued for nine months.

\section{Clinical examination included:}

Periodontal status: Assessed on six sextants/ subject according to Loe and Silness ${ }^{(18)}$ for gingival index, plaque index and $\mathrm{WHO}^{(19)}$ for DMF.

Gingival Index: a numerical rating scale for classifying the periodontal status of a person or population with a single figure which take into consideration prevalence as well as severity of the condition. It is based upon probe measurement of periodontal pockets and on gingival tissue status. In this study clinical assessment includes only the gingival status. A graded assessment of gingival health used in periodontal charting to evaluate a case of gingivitis based on visual inspection of the gingiva that takes into consideration the color and firmness of gingival tissue along with the presence of blood during probing.

Gingival index system as follows:

\begin{tabular}{|c|c|c|c|}
\hline Appearance & bleeding & inflammation & points \\
\hline Normal & No bleeding & none & 0 \\
\hline $\begin{array}{c}\text { Slight change in color } \\
\text { and mild edema with } \\
\text { slight change in texture }\end{array}$ & No bleeding & mild & 1 \\
\hline $\begin{array}{c}\text { Redness, hypertrophy } \\
\text {,edema, glazing }\end{array}$ & $\begin{array}{c}\text { Bleeding on } \\
\text { probing/pressure }\end{array}$ & Moderate & 2 \\
\hline $\begin{array}{c}\text { Marked redness, } \\
\text { hypertrophy, edema, } \\
\text { ulceration }\end{array}$ & $\begin{array}{c}\text { Spontaneous } \\
\text { bleeding }\end{array}$ & sever & 3 \\
\hline
\end{tabular}

\section{Gingival index mean calculation}

The index for each volunteer is obtained by summing the indices for all six teeth and dividing by six

Plaque index: Is the measurement of the state of oral hygiene by loe \& Silness. It is based on recording both soft debris and mineralized deposit, and considered as an assessment tool to evaluate the thickness of the plaque at the gingival margin that may be applied to selected teeth or to the entire oral cavity.

Plaque index system as follows:

\begin{tabular}{|c|c|}
\hline scores & criteria \\
\hline 0 & No plaque \\
\hline 1 & $\begin{array}{c}\text { A film of plaque adhering to the free gingival margin and } \\
\text { adjacent area of the tooth. The plaque may be seen in situ } \\
\text { only after application of disclosing solution or by using the } \\
\text { probe on tooth surface }\end{array}$ \\
\hline 2 & $\begin{array}{c}\text { Moderate accumulation of soft deposit within the gingival } \\
\text { pocket, or the tooth and gingival margin which can be seen } \\
\text { by the naked eye }\end{array}$ \\
\hline 3 & Abundance of soft matter on the tooth and gingival margin. \\
\hline
\end{tabular}

Plaque index calculation: The index of the subject was obtained by summing the indices for all six teeth and dividing by six.

Disclosing agent: in the form of tablets colored (red-cote dent disclosing tabs. butler Code No 130065-0), examination was performed after chewing one tablet where supra gingival plaque could be seen as a violet or purple spots.

Sites of examination: The teeth examined in GI and PI represented six sites and 4 surfaces on each tooth: 1) buccal, 2) lingual, 3) mesial, 4) distal

1) Maxillary right 1 st molar (16)

2) Maxillary right lateral incisor (12)

3) Maxillary left 1 st bicuspid (24)

4) Mandibular left 1st molar (36)

5) Mandibular left lateral incisor (32)

6) Mandibular right 1 st bicuspid (44)

Missing teeth were not substituted.

DMF index: DMF index has been recommended by WHO, as a parameter to assess the dental health situation in various societies. It has also been shown that DMF index is directly correlated with the health \& economic, cultural, social state of the society and therefore it can be used for comparative studies $^{(121)}$.

\section{DMF index calculation}

The DMF index mean was calculated simply as the total number of decayed teeth (D), missing (M) and filled (F) teeth divided by the number of cases investigated. Great variations between societies, has been noted.

Statistical analysis: The data has been organized and analyzed using the statistical program SPSS (Statistical Packages for Social Sciences): the T-test for equality of means; Regressions and curve estimations for relationships between different parameters; Descriptive statistics included cross-tabulations and simple charts.

Ethical Clearance: The research protocol has been approved by Research Ethical Committee, Faculty of Dentistry, Khartoum University. All participating subjects 


\section{International Journal of Science and Research (IJSR) \\ ISSN (Online): 2319-7064 \\ Index Copernicus Value (2015): 78.96 | Impact Factor (2015): 6.391}

signed a standardized informed consent form as a prerequisite to their participation.

No Conflict of Interest Reported.

\section{Results}

Variations in DMF, PI and GI index when related to brushing tool texture:

An interesting relationship was observed between oral hygiene measure indices and texture of the tooth brush texture [Table.1].

Table 1: Comparing the effect of brushing tool texture on DMF, PI and GI

\begin{tabular}{|c|c|c|c|c|c|c|c|c|c|}
\hline $\begin{array}{c}\text { Oral Hygiene } \\
\text { measures }\end{array}$ & DMF & No & Mean \pm SD & PI & No & Mean \pm SD & GI & No & Mean \pm SD \\
\hline \multirow[t]{5}{*}{ Texture Type } & \multirow{2}{*}{ Soft } & \multirow[t]{2}{*}{19} & \multirow{2}{*}{$6.22 \pm 4.89$} & \multirow{2}{*}{ Soft } & \multirow{2}{*}{19} & $1.56 \pm 0.78$ & Soft & 18 & $1.72 \pm 0.5$ \\
\hline & & & & & & & Hard & 13 & $1.31 \pm 0.4$ \\
\hline & \multirow{2}{*}{ Hard } & \multirow[t]{2}{*}{14} & \multirow{2}{*}{$2.85 \pm 2.48$} & \multirow{2}{*}{ Hard } & \multirow{2}{*}{14} & $1.38 \pm 0.50$ & Soft & 18 & $1.31 \pm 0.4$ \\
\hline & & & & & & & Medium & 24 & $1.72 \pm 0.57$ \\
\hline & Medium & 24 & $5.29 \pm 4.02$ & medium & 26 & $1.21 \pm 0.51$ & & & $1.21 \pm 0.41$ \\
\hline P-value & & & 0.077 & & & 0.199 & & & $\frac{0.023 *}{0.001 * *}$ \\
\hline
\end{tabular}

Significant differences were detected in the oral hygiene measure GI index when related to the three textures of the tooth paste brush bristles with a p-value $=0.001$ between soft and medium and 0.023 between soft and hard. High gingival index was shown with the soft texture in [Fig.1].

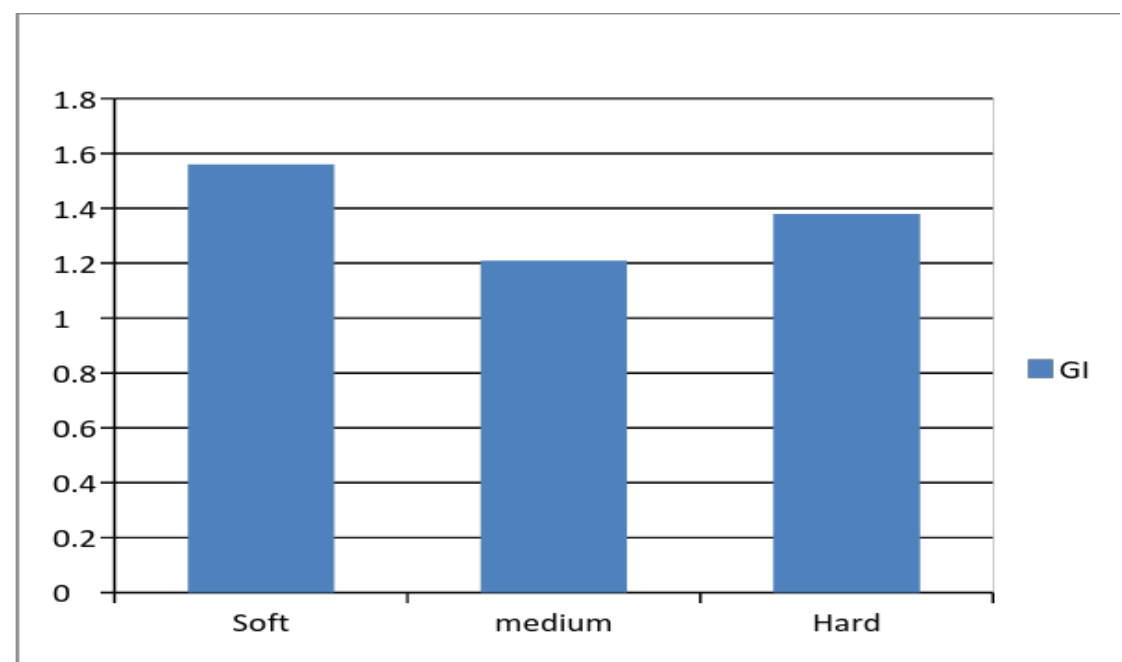

Figure 1: Relationship of the GI index to tooth brush texture

Although PI index p-value was insignificant in the three textures, yet the study revealed a significant difference ( $\mathrm{p}$ value $=0.001$ ) between soft and medium texture [Fig .2].

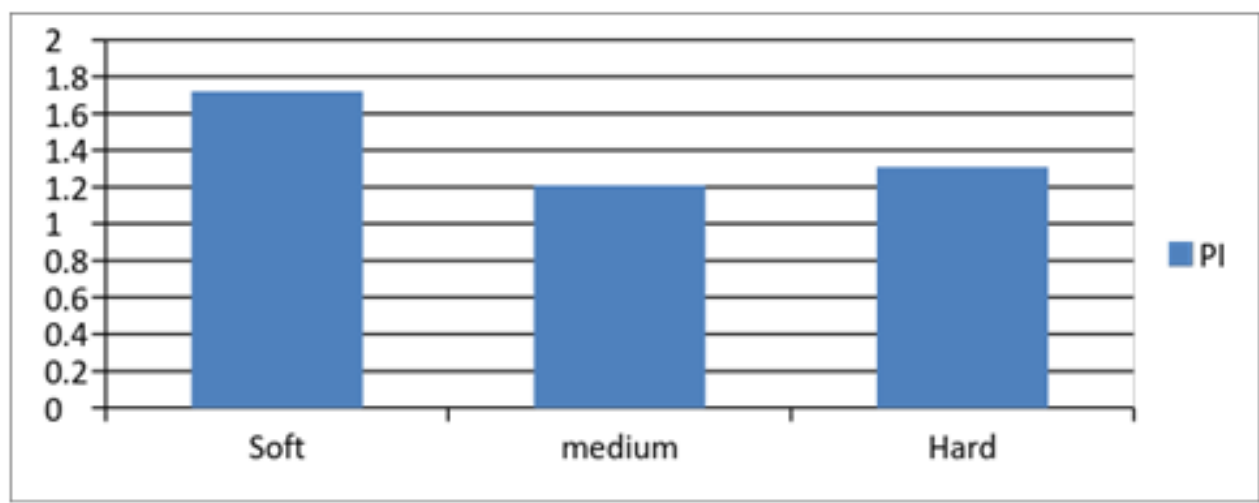

Figure 2: Relationship of the PI index to the texture of the tooth brush

Volume 6 Issue 7, July 2017 www.ijsr.net 


\section{International Journal of Science and Research (IJSR) \\ ISSN (Online): 2319-7064}

Index Copernicus Value (2015): 78.96 | Impact Factor (2015): 6.391

\section{Discussion}

Before the invention of the toothbrush a variety of oral hygiene measures had been used for nearly 5000 years ${ }^{(1)}$. This has been verified by excavations in which chew sticks, tree twigs, bird feathers, animal bones and porcupine quills were recovered ${ }^{(20)}$. Chew sticks were twigs with frayed ends used to brush the teeth ${ }^{(21)}$, while the other end was used as a toothpick ${ }^{(22)}$. The earliest chew sticks were discovered in Babylonia in $3500 \mathrm{BC}$, bristle toothbrush resembling the modern one was first reported in China during the Tang Dynasty (619-907), consisting of bristles that were sourced from hogs living in Siberia and northern China as colder temperatures provided firmer bristles, attached to a handle manufactured from bamboo or bone, forming a toothbrush ${ }^{(21)}$. The bristle toothbrush spread to Europe from China to Europe by travelers ${ }^{(22)}$, where it was adopted during the 17 th century ${ }^{(23)}$. The earliest identified use of the word toothbrush in English was in the autobiography of Anthony Wood who wrote in 1690 that he had bought a toothbrush from J. Barret ${ }^{(24)}$. During the 1900 s, celluloid gradually replaced bone handles ${ }^{(25)}$, and natural animal bristles were also replaced by synthetic fibers, usually nylon, by DuPont in 1938. By the turn of the 21 st century nylon had come to be widely used for the bristles, handles were usually molded from thermoplastic materials ${ }^{(2)}$. In January 2003 the toothbrush was selected as the number one invention, Americans could not live without according to the Lemelson-MIT Invention Index ${ }^{(26)}$. But industry has flooded the markets with different types of toothbrushes and different brittle textures, which necessitated more research and data on their efficacy. Our study comes within this domain. The comparison of the different types of tooth brushes (soft, medium and hard) in this study revealed a significant relationship with periodontal gingival index. The least, and best index was found to be with the medium type of brush, while the soft brush showed the highest and worst gingival index. Literature is scanty, in a previous study conducted at Franciscan University, Santa Maria Brazil, researchers recommended, and contrary to our findings for a different reason, short rounded ended medium bristles for effective mechanical daily removal of dental plaque prevent periodontal diseases, dental caries and gingivitis ${ }^{(27) .}$ In that study which compared the difference in medium and soft toothbrush bristles for plaque removal and soft-tissue abrasion, a total of 25 undergraduate students free from gingivitis at the onset of the study, were asked to refrain from all oral hygiene for 96 hours, to allow plaque to accumulate. Students were then_randomly assigned to brush two quadrants of their mouth with the medium bristle toothbrush, and the other two quadrants with the soft brush. The lower quadrants were brushed with Colgate Triple Action toothpaste and the upper quadrants were brushed without toothpaste. The results revealed that medium toothbrushes removed high amounts of dental biofilm compared to soft toothbrushes $(\mathrm{P}<0.05)$, but there was a higher proportion of abrasions in the medium toothbrush group $(\mathrm{P}<0.01)$ particularly in the cervical regions, thus concluding that though medium toothbrushes have a greater ability to remove biofilm, they cause more gingival abrasion than do soft toothbrushes, so favoring soft toothbrushes. In our study we haven't assessed that parameter of gingival abrasions. There are, however, still more limitations that faced our study, to name deficiency in proper methodological guidelines, standardization of design, absence of consensus criteria, as well inconclusive evidence in literature. All call for further research in this enormously expanding industry.

\section{Conclusion}

Medium bristles of the tooth brush were found to be more effective and better for oral hygiene than soft or hard tooth brush bristles, regardless of the type of the tooth brush. Considering the mentioned limitations, there is high need to do further research on the how toothbrush texture brittleness affect oral hygiene.

\section{References}

[1] Lindhe J, Axelsson P, Tollskog G. Effect of proper oral hygiene on gingivitis. And dental caries in Swedish schoolchildren Community. Dental Oral Epidemiol. 1975; 3: 150-155

[2] Lindhe J, Axelsson P, Tollskog G. Effect of proper oral hygiene on gingivitis and dental caries in Swedish schoolchildren. Community Dent Oral Epidemiol. $1975 ; 3: 150-5$.

[3] Dale JW. Toothbrushing frequency and its relationship to dental caries and periodontal disease. Aust Dent J. $1969 ; 14: 120-3$.

[4] Tucker GJ, Andlaw RJ, Burchell CK. The relationship between oral hygiene and dental caries incidence in 11year-old children. A 3-year study. Br Dent J. 1969; 141:75-9.

[5] Ainamo J. Current basis for the prevention of dental caries. J Indian Dent Assoc. 1969; 48:117-24.

[6] Claydon N, Addy M. Comparative single-use plaque removal by toothbrushes of different designs. J Clin Periodontol. 1996; 23:1112-6.

[7] Loe H, Theilade E, Jensen SB. Experimental gingivitis in man. J Periodontol. 1965; 36:177-87.

[8] Frandsen A. Mechanical oral hygiene practices. In: Löe H, Kleinman DV, editors. Dental Plaque Control Measures and Oral Hygiene Practices. Oxford, Washington, DC: IRL Press; 1986; 93-116.

[9] Cifcibasi E, Koyuncuoglu CZ, Baser U, Bozacioglu B, Kasali K, and Cintan S. Comparison of manual toothbrushes with different bristle designs in terms of cleaning efficacy and potential role on gingival recession. Eur J Dent. 2014; 8(3): 395-401.

[10]Ren YF, Cacciato R, Whelehan MT, Ning L, Malmstrom HS. Effects of toothbrushes with tapered and cross angled soft bristle design on dental plaque and gingival inflammation: A randomized and controlled clinical trial. J Dent. 2007; 35:614-22.

[11] Jepsen $\mathrm{S}$. The role of manual toothbrushes in effective plaque control: Advantages and limitations. In: Lang NP, Attstrom R, Löe H, editors. Proceedings of the European Workshop on Mechanical Plaque Control. London: Quintessence; pp. 121-37. 8. Sammons, R. (2003). "Control of dental plaque". Medical biofilms detection, prevention and control. Chichester: John Wiley \& Sons. 1998; 223.

\section{Volume 6 Issue 7, July 2017 www.ijsr.net}


[12] Beals D, Ngo T, Feng Y, Cook D, Grau DG, Weber DA. Development and laboratory evaluation of a new toothbrush with a novel brush head design. Am J Dent. $13: 5 \mathrm{~A}-1$

[13] Sharma NC, Qaqish J, Walters PA, Grender J, Biesbrock AR (2010). A clinical evaluation of the plaque removal efficacy of five manual toothbrushes. $\mathbf{J}$ Clin Dent. 2000; 21:8-12.

[14] Stiller S, Bosma ML, Shi X, Spirgel CM, Yankell SL. Interproximal access efficacy of three manual toothbrushes with extended, $\mathrm{x}$-angled or flat multitufted bristles. Int J Dent Hyg. 2010; 8:244-8.

[15]Zimmer, S; Öztürk, M; Barthel, CR; Bizhang, M; Jordan, RA. "Cleaning efficacy and soft tissue trauma after use of manual toothbrushes with different bristle stiffness". J Periodontol. 2011; 82 (2): 267-71].

[16] "Oral Longevity," American Dental Association brochure (PDF), page 2 Retrieved June 12, 2008. Oral Health Prev Dent. 2011; 9(2):177-83.

[17] Loe H, Theilade E, Jensen SB. Experimental gingivitis in man. J Periodontol. 1965; 36:177-87.

[18] Loe H. The Gingival Index, the Plaque Index, and the Retention Index. J Periodontol. 1967; 38: 610-616.

[19] World Health Organization. Oral health surveys. Basic methods. Geneva. WHO, 1987.

[20] Sammons, R. "Control of dental plaque". Medical biofilms detection, prevention and control. Chichester: John Wiley \& Sons. 203; p. 223. ISBN 978-0-47198867-0.

[21] Panati, Charles. Extraordinary Origins of Everyday Things. HarperCollins. 2013; pp. 208-209.

[22] Yu, Hai-Yang; Qian, Lin-Mao; Zheng, Jing. Dental Biotribology. Springer. 2013; pp. 18-19. ISBN 978-14614-4550-0.

[23]"Who invented the toothbrush and when was it invented?". The Library of Congress. 2007. Retrieved 2008; 04-12.

[24] Stay, Flora Parsa. The fibromyalgia dental handbook: A practical guide to maintaining peak dental health. New York: Marlowe \& Company. 2005; p. 118. ISBN 9781-56924-401-2.

[25] Olmert, Michael. Milton's teeth \& Ovid's umbrella: curiouser and curiouser adventures in history. New York: Simon \& Schuster. 1996; p. 62.

[26] Kumar, Jayanth V. "Oral hygiene aids". Textbook of preventive and community dentistry (2nd ed.). Elsevier. 2011; pp. 412-413.

[27]"2003 Invention Index". 2003-01-21. Retrieved 200905-20.

[28]Zanatta FB1, Bergoli AD, Werle SB, Antoniazzi RP. Biofilm removal and gingival abrasion with medium and soft toothbrushes. Oral Health Prev Dent. 2011; 9(2):177-83.

\section{Author Profile}

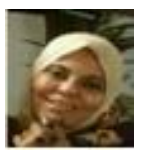

Dr Wafaa Abdulraouf Khalil is a MBBS, $\mathrm{PhD}$ Human Physiology and Assistant Professor, Faculty of Dentistry, Khartoum University, Khartoum Sudan. 